Chirurg 2022 $93: 64-71$

https://doi.org/10.1007/s00104-021-01547-x Angenommen: 8. November 2021 Online publiziert: 10. Dezember 2021

(C) The Author(s), under exclusive licence to Springer Medizin Verlag GmbH, ein Teil von Springer Nature 2021

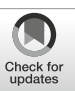

\section{Ressourcenbedarf bei der chirurgischen Behandlung von COVID-19-Patienten in der universitären Maximalversorgung}

\author{
H. Kerndl' · D. Liebetrau' · S. Zerwes ${ }^{1}$ - C. Römmele ${ }^{2}$ A. Hyhlik-Dürr' ${ }^{1}$ \\ ${ }^{1}$ Gefäßchirurgie, Medizinische Fakultät, Universitätsklinikum Augsburg, Augsburg, Deutschland \\ ${ }^{2}$ III. Medizinische Klinik, Universitätsklinikum Augsburg, Augsburg, Deutschland
}

Zusammenfassung

Hintergrund: Operative Eingriffe bei an "coronavirus disease 2019" (COVID-19) erkrankten Patienten sind unter strengen Hygiene- und Schutzmaßnahmen möglich und werden inzwischen regelhaft durchgeführt. In dieser Arbeit soll geprüft werden, mit welchem Mehraufwand dies verbunden ist.

Material und Methoden: Es erfolgte die strukturierte Auswertung von 71 am Universitätsklinikum Augsburg zwischen dem 01.11.2020 und 31.12.2020 durchgeführten Operationen. Weiterhin erfolgte der Vergleich von zeitlichem, strukturellem und personellem Aufwand zwischen Operationen an COVID-19Patienten und Non-COVID-19-Patienten am Beispiel von vier Eingriffen: transbrachiale Embolektomie, Implantation von Hüfttotalendoprothesen (Hüft-TEP) oder Proximalem Femurnagel Antirotation (PFN-A) und Herzschrittmachern.

Ergebnisse: Die Schnitt-Naht-Zeiten zwischen den Eingriffen bei an COVID-19Erkrankten und Non-COVID-Patienten zeigten bei keinem der vier untersuchten Eingriffe signifikante Unterschiede. Die bei vielen Eingriffen als zeitaufwendig identifizierte postoperative Überwachung im Operationssaal wurde häufig durch postoperative Verlegung auf Intensivstation oder durch die Verwendung lokaler Anästhesieverfahren umgangen. Bei großen Operationen wie Hüft-TEP-Implantation war die Vorbereitungszeit signifikant verlängert. $(p=0,037)$. Des Weiteren zeigte sich ein signifikant höherer Bedarf an Anästhesiepflegekräften (1,5 vs. 1,0, $p=0,02$ ). Schlussfolgerung: Eine Quantifizierung des Mehraufwandes der operativen Versorgung ist durch die ohnehin komplexe Versorgung der COVID-19-Patienten nur schwer möglich. Allerdings ist davon auszugehen, dass ein erhöhter, durch die Standarderfassung nicht dokumentierter Bedarf an zusätzlichen personellen und strukturellen Ressourcen durch die Materialzubringung von außerhalb des Operationssaales besteht.

\section{Studientyp}

Retrospektive Auswertung aller am Universitätsklinikum Augsburg durchgeführten Operationen an COVID-19-Patienten zwischen 01.11.2020 und 31.12.2020

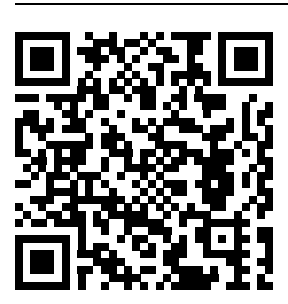

QR-Code scannen \& Beitrag online lesen

\title{
Schlüsselwörter
}

Operationsmanagement · Transbrachiale Embolektomie · Hüfttotalendoprothese · Proximaler Femurnagel Antirotation · Herzschrittmacher

\section{Hintergrund}

Die Coronavirus(„,severe acute respiratory syndrome coronavirus type $2^{\prime \prime}$ [SARS CoV2], ,"coronavirus disease 2019" [COVID-19])Pandemie stellt medizinische Gesundheitseinrichtungen vor große logistische, personelle und organisatorische Heraus- forderungen. Am Universitätsklinikum Augsburg (UKA) wurde zur Sicherstellung ausreichender Kapazitäten für die operative Versorgung von COVID-19-Patienten ein weiterer Operationssaal ausschließlich für COVID-19-Patienten geschaffen.

Da auf rein elektive Eingriffe bei Corona-Patienten größtenteils verzichtet wird 


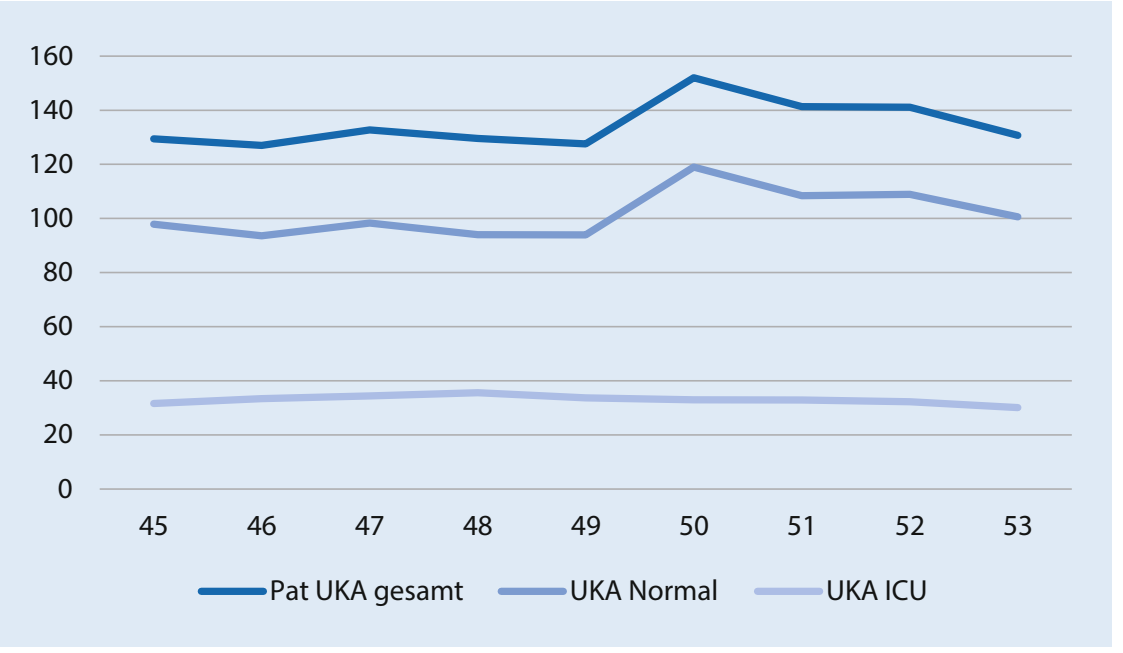

Abb. 1 A Anzahl der stationär behandelten COVID-19 ("coronavirus disease 2019")-Patienten am UKA im betrachteten Zeitraum von Kalenderwoche 45 bis 53, Unterteilung in Gesamtanzahl der Patienten, Patienten auf Normalstation und Patienten auf Intensivstation. X-Achse Zahl der stationär behandelten Patienten am UKA, Y-Achse Kalenderwochen 2020. ICU "intensive care unit", Pat Patient, UKA Universitätsklinikum Augsburg

[8], handelt es sich bei den durchgeführten Operationen um nicht aufschiebbare oder dringlich indizierte Eingriffe. Neben den notwendigen Hygienemaßnahmen im Umgang mit COVID-19-Patienten bedarf es bei Planung und Durchführung von Operationen an COVID-19-Patienten weiterer Überlegungen [1]. Diese lassen sich in technisch strukturelle, personelle sowie generell organisatorisch strukturelle unterteilen. In diesem Beitrag erfolgten die Betrachtung der Strukturen am Universitätsklinikum Augsburg und die operative Versorgung von 71 COVID-19-Patienten im Rahmen der zweiten COVID-19Welle in Deutschland. Außerdem erfolgte ein Vergleich des zeitlichen sowie personellen Aufwandes bei Operationen am Beispiel von Eingriffen bei COVID-19- und Non-COVID-Patienten.

Im Herbst 2020 kam es im Rahmen der zweiten Infektionswelle der COVID-19Pandemie zu einem starkem Patientenzustrom an COVID-19 erkrankten Patienten (- Abb. 1). Mit steigender Anzahl an Infektionen und Verdachtsfällen erhöhte sich auch die Notwendigkeit von Operationen an COVID-19 erkrankten Patienten. Insbesondere Patienten mit dringlicher oder notfallmäßiger Indikation müssen trotz positiver Testung oder ohne Ausschluss der Infektion durch negative Testung bei Verdachtsfällen auf das SARS-CoV-2 operativ versorgt werden. Die Durchführung

\section{Patientenkollektiv und Methoden}

Betrachtet wurden alle durchgeführten Operationen an Patienten mit bestätigter Infektion (einmalige positiver PCR[,polymerase chain reaction“]-Test ohne Beendigung der Quarantäne oder folgende negative Testungen) oder mit Verdacht auf eine COVID-19-Infektion (klinisch, positiver Schnelltest, Quarantäne als COVID-19-Kontaktperson) im Zeitraum vom 01.11.2020 bis 31.12.2020 am Universitätsklinikum Augsburg (UKA).

Alle Operationen wurden in einem speziell für COVID-19-Patienten etablierten und betriebenen Operationssaal durchgeführt. Dieser ist am UKA als spezieller septischer Operationssaal nach Vorgaben der Deutschen Gesetzlichen Unfallversicherung etabliert und verfügt somit bereits über die nötigen baulichen Vorrausetzungen [4]. Das Betreten erfolgt über eine separate Umkleide mit direktem Zugang über einen eigenen Waschbereich. Das Einschleusen des Patienten in den Operationssaalbereich erfolgt über eine abgetrennte Schleuse ohne Kontakt zur Patientenholding oder zum Aufwachraum. Der Transport zum Operationssaal erfolgt durch speziell geschultes Personal mit Vollkontaktschutzkleidung und über separat nutzbare Aufzüge. Somit findet kein Kontakt des COVID-19-Patienten zum Krankenhausbetrieb und zum regulären Operationssaaltrakt statt. Die Narkoseeinleitung wird immer direkt im Operationssaal und nicht in den regulär genutzten speziellen Narkoseeinleitungseinheiten vorgenommen.

Die verbaute raumlufttechnische Anlage erzeugt einen kontinuierlichen Unterdruck im Operationssaal. Dieser beträgt bei geschlossenen Türen -2/-6 Pa. Außerdem findet neben einer Luftzirkulation über ein Filtersystem eine kontinuierliche Luftfilterung statt. Zusätzlich werden pro Stunde $717 \mathrm{~m}^{3}$ als Abluft abgeführt. In den nichtseptischen/Non-COVID-Operationssälen besteht im Vergleich zu den umgebenden Räumen ein positiver Luftdruck und die stündliche Abluftmenge ist mit $433 \mathrm{~m}^{3}$ deutlich geringer, dies entspricht der DIN 1946-4, die die Lüftungsanlagen in Operationsräumen regelt [11].

Zur Analyse der durchgeführten Operationen erfolgte eine retrospektive Auswer- 


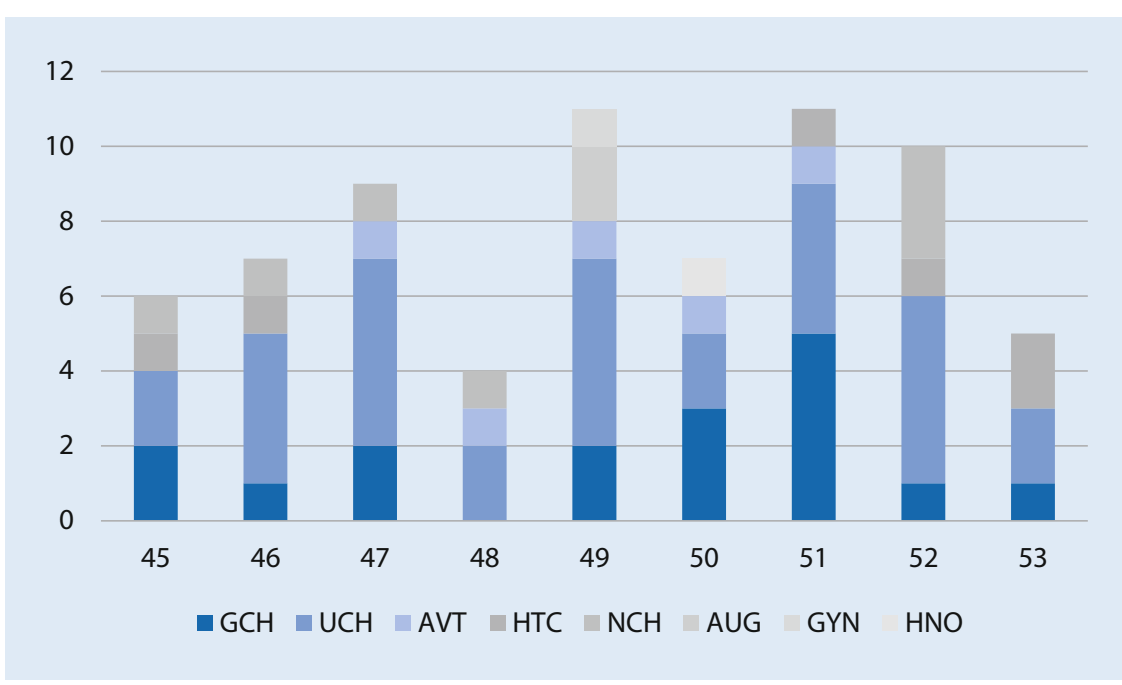

Abb. $2 \Delta$ Anzahl der durchgeführten Operationen an COVID-19-Patienen am Universitätsklinikum Augsburg in den Kalenderwochen 45 bis 53 im Jahr 2020 mit Darstellung der unterschiedlichen Fachabteilungen. $X$-Achse Zahl der pro Woche durchgeführten Operationen, $Y$-Achse Kalenderwochen 2020. AUG Augenheilkunde, AVT Allgemeine, Viszeral- und Transplantationschirurgie, GCH Gefäßchirurgie, GYN Gynäkologie, HNOHals-Nasen-Ohren-Chirurgie, HTCHerz-Thorax-Chirurgie, NCH Neurochirurgie, UCHUnfallchirurgie

tung der Operationsdaten und Patientencharakteristika durch Sichtung des Klinikpatientenmanagementsystems Orbis (Dedalus HealthCare, Konrad-Zuse-Platz 1-3, Bonn) und des lokal geführten COVID-19Registers (COKA-Register, Universitätsklinikum Augsburg, Augsburg, Deutschland) des UKA mit Auflistung von am UKA behandelten COVID-19-Patienten. Patientencharakteristika wie Geschlecht, Alter, BodyMass-Index (BMI), ASA(American Society of Anesthesiologists)-Klassifikation, Dauer des stationären Aufenthalts, Intensivaufenthalt, invasive Beatmung und Vorerkrankungen wurden systematisch erfasst und retrospektiv in eine Datenbank (Microsoft Excel 2020, One Microsoft Way, Redmond, WA, USA) überführt. Ebenso wurden die durchgeführten Eingriffe in Bezug auf Dringlichkeit des Eingriffes (vitaler Notfall, dringlich [ $<6 / 24 \mathrm{~h}]$ oder elektiv), Anästhesieverfahren, beteiligtes Personal und Operationsdauer analysiert. Bei der Operationsdauer erfolgte eine selektive Betrachtung der Vorbereitungsdauer vom Beginn der Operationsvorbereitung im Operationssaal bis zum Schnitt, der Schnitt-NahtZeit und der Gesamtoperationsdauer von der Vorbereitung bis zum Ausschleusen des Patienten aus dem Operationssaal. Bei der Betrachtung des beteiligten Personals wurden alle Mitarbeiter, die im Operationsprotokoll aufgeführt wurden, berücksich- tigt. Die Studie wurde im Einklang mit der Deklaration von Helsinki und dem "good clinical practice" durchgeführt. Eine positive Begutachtung durch die Ethikkommission für das COKA-Register erfolgte unter der Eingangsnummer 20-426. Eine Begutachtung für die gesamte Studie erfolgte unter der Eingangsnummer 21-0805.

Für die vier am häufigsten durchgeführten Eingriffe (Armembolektomie, Hüfttotalendoprothesen[Hüft-TEP]-Implantation, Implantation Proximaler Femurnagel Antirotation [PFN-A], Herzschrittmacherneuimplantation) erfolgte eine systematische Auswertung der Operationsdaten (Vorbereitungszeit, Schnitt-Naht-Zeit, Gesamtdauer sowie Analyse des beteiligten Personals). Anschließend wurde eine retrospektive Kontrollgruppe aus Non-COVIDPatienten erstellt, die im selben Zeitraum oder bei geringerer Eingriffsanzahl im Jahr 2020 denselben Eingriff (identische OPS[Operationen- und Prozedurenschlüssel]-Codes) erhalten hatte. Dabei wurde speziell auf dieselben OPS-Codes geachtet. Ein gezieltes Matching nach weiteren Patientencharakteristika fand bei geringen Patientenzahlen nicht statt. Die durchgeführten Operationen wurden ebenso hinsichtlich der Patientencharakteristika (Geschlecht, Alter, Vorerkrankungen) sowie der Operationszeiten und des benötigten Personals analysiert und die Mittel- werte sowie Standardabweichungen der Operationsdaten bestimmt. Abschließend erfolgte die statistische Analyse mittels SPSS (Version 27, IBM Deutschland, Ehningen, Deutschland) auf signifikante Unterschiede zwischen den Gruppen.

\section{Ergebnisse}

Der Untersuchungszeitraum wurde im Rahmen der zweiten COVID-19-Erkrankungswelle in Deutschland im Herbst 2020 gewählt. Wie aus D Abb. 1 hervorgeht, kam es in den Kalenderwochen (KW) 49 bis 51 zu einem deutlichen Zustrom an COVID-19-positiven Patienten in das Universitätsklinikum Augsburg.

In $\square$ Abb. 2 ist der Verlauf der durchgeführten Operationen an COVID-19-Patienten im betrachteten Zeitraum mit höchstens 11 durchgeführten Operationen in KW 49 und 51 dargestellt. Es wurden maximal 4 Patienten an einem Tag im betrachteten speziellen COVID-Operationssaal operiert.

Im untersuchten Zeitraum wurden 71 Operationen an COVID-19-Patienten oder an Patienten mit möglicher COVID-19-Infektion durchgeführt. Es wurden 37 Männer und 34 Frauen operiert. Das durchschnittliche Alter betrug 66,5 Jahre (Median 73 Jahre). Der BMI der untersuchten Patienten lag im Mittel bei $29,5 \mathrm{~kg} / \mathrm{m}^{2}$. Die durchschnittliche Krankenhausaufenthaltsdauer lag bei 28,3 Tagen. 24 Patienten wurden aufgrund der COVID-19Infektion auf Intensivstation behandelt, 14 davon wurden bereits präoperativ auf Intensivstation behandelt, bei 9 (17\%) Patienten wurde eine COVID-19-assoziierte invasive Beatmung notwendig.

Zum Ende des Beobachtungszeitraums am 31.12.2020 befanden sich 19 Patienten noch in stationärer Behandlung, 38 wurden entlassen und 14 Patienten sind im Krankenhaus verstorben, dies entspricht einer Gesamtletalität von $22 \%$ im Kollektiv der betrachteten Patienten im beobachteten Zeitraum. - Tab. 1 zeigt die Patientencharakteristika der behandelten COVID-19-Patienten.

Die Verteilung und Art der Operationen mit Betrachtung der einzelnen Fachdisziplinen zeigt $\bullet$ Tab. 2.

Bei den vier am häufigsten durchgeführten Operationen an COVID-19-positi- 
Tab. 1 Patientencharakteristika der operativ behandelten COVID-19-Patienten COVID-19-Patienten

\begin{tabular}{|l|l|l|}
\hline \multirow{2}{*}{} & \multicolumn{2}{|l|}{$\begin{array}{l}\text { COVID-19-Pati- } \\
\text { enten }\end{array}$} \\
\cline { 2 - 3 } & $(n)$ & $(\%)$ \\
\hline Einbezogene Patienten & 71 & 100 \\
\hline Geschlecht (m/w) & $37 / 34$ & $52,1 / 47,9$ \\
\hline Alter (Jahre) & $66,5 \pm 18,5$ \\
\hline Medianes Alter (Jahre) & 73 \\
\hline Body-Mass-Index & $29,5 \pm 10,4$ \\
\hline $\begin{array}{l}\text { Krankenhausaufenthalt } \\
\text { (Tage) }\end{array}$ & $28,3 \pm 30,6$ \\
\hline $\begin{array}{l}\text { Aufenthalt auf Inten- } \\
\text { sivstation }\end{array}$ & 24 & 33,8 \\
\hline Invasive Beatmung & \multicolumn{2}{|l|}{} \\
\hline Vorerkrankungen & 9 & 12,7 \\
\hline Arterielle Hypertonie & 45 & 63,4 \\
\hline Diabetes & 4 & 5,6 \\
\hline Koronare Herzkrankheit & 16 & 22,5 \\
\hline $\begin{array}{l}\text { Tiefe Venenthrombose } \\
\text { in Anamnese }\end{array}$ & 2 & 2,8 \\
\hline $\begin{array}{l}\text { Lungenarterienembolie } \\
\text { in Anamnese }\end{array}$ & 4 & 5,6 \\
\hline Niereninsuffizienz & 18 & 12,7 \\
\hline Klinisches Outcome & 14 & 19,7 \\
\hline Aktuell im Krankenhaus & 19 & 26,8 \\
\hline Entlassen & 38 & 53,5 \\
\hline Tod & \multicolumn{2}{|l}{} \\
\hline${ }^{2}$ Notwendig während des Aufenthalts \\
\hline
\end{tabular}

ven Patienten (13) handelte es sich um die Versorgung mittels PFN A (4), Hüft-TEP (3), Herzschrittmacherneuimplantation (4) und transbrachiale Embolektomien (3). Im gewählten Zeitraum wurden an NonCOVID-Patienten 23 Herzschrittmacherneuimplantationen, 19 PFN-A, 13 HüftTEPs und 8 transbrachiale Embolektomien durchgeführt. Das durchschnittliche Alter der COVID-19-Patienten lag bei 82 Jahren, dass der Non-COVID-Patienten bei 81 Jahren (• Tab. 3).

Bei $61,5 \%$ der Operationen an COVID -19-Patienten lag eine dringliche Operationsindikation innerhalb der nächsten $24 \mathrm{~h}$ vor, (Non-COVID-Patienten $63 \%$ ), zusätzlich wurden $5 \%$ der Operationen an NonCOVID-Patienten als Notfall im Operationssystem hinterlegt, bei den COVID-19-Patienten wurde diese Art der Dringlichkeit nicht vermerkt.

Bei den verwendeten Anästhesieverfahren zeigten sich keine signifikanten Unterschiede, in 53,8\% der Fälle erfolgte der Eingriff an COVID-19-Patienten in Intuba-
Tab. 2 Auflistung der durchgeführten Eingriffe und Verteilung auf die verschiedenen operativen Fachabteilungen

\begin{tabular}{|c|c|c|}
\hline \multirow[t]{2}{*}{ Fachbereich } & \multicolumn{2}{|c|}{$\begin{array}{l}\text { COVID-19- } \\
\text { Patienten }\end{array}$} \\
\hline & $(n)$ & (\%) \\
\hline Einbezogene Patienten & 71 & 100 \\
\hline Unfallchirurgie & 32 & 45,1 \\
\hline Weichteileingriffe & 12 & - \\
\hline Osteosynthesen & 14 & - \\
\hline Endoprothesen & 6 & - \\
\hline Gefäßchirurgie & 18 & 25,4 \\
\hline Arterielle Rekonstruktionen & 5 & - \\
\hline Weichteileingriffe & 10 & - \\
\hline Amputationen & 2 & - \\
\hline Sonstige & 1 & - \\
\hline $\begin{array}{l}\text { Allgemeine, Viszeral- und } \\
\text { Transplantationschirurgie }\end{array}$ & 5 & 7,0 \\
\hline Laparoskopisch $^{\mathrm{a}}$ & 2 & - \\
\hline Offen chirurgisch ${ }^{a}$ & 1 & - \\
\hline Weichteileingriffe & 1 & - \\
\hline Sonstige & 1 & - \\
\hline Herz-Thorax-Chirurgie & 6 & 8,5 \\
\hline $\begin{array}{l}\text { Herzschrittmacher- } \\
\text { neuimplantation }\end{array}$ & 4 & - \\
\hline Sonstige & 2 & - \\
\hline Hals-Nasen-Ohren-Chirurgie & 1 & 1,4 \\
\hline Tracheotomie & 1 & - \\
\hline Augenheilkunde & 2 & 2,8 \\
\hline Operation bei Ablatio retinae & 2 & - \\
\hline Gynäkologie & 1 & 1,4 \\
\hline Abrasio & 1 & - \\
\hline Neurochirurgie & 6 & 8,5 \\
\hline Ventrikeldrainage & 2 & - \\
\hline Intrakranielle Blutung & 2 & - \\
\hline Sonstige & 2 & - \\
\hline${ }^{a}$ Abdomineller Eingriff & & \\
\hline
\end{tabular}

tionsnarkose (Non-COVID-Patienten 52\%), in $7,7 \%$ mit Atemwegssicherung mittels Larynxmaske (Non-COVID-Patienten 8\%). $38,5 \%$ der Eingriffe an COVID-19-Patienten erfolgten in Regional- oder Lokalanästhesie (Non-COVID-Patienten $40 \%$ ).

Bei Betrachtung des beteiligten Personals zeigte sich in der Anzahl der beteiligten operativen Ärzte kein Unterschied zwischen COVID-19-Patienten und Non-COVID-Patienten (durchschnittlich 1,9 vs. 1,8). Beim erfassten operativen Pflegepersonal zeigt sich mit 2,2 vs. 2,7 (COVID/Non-COVID) beteiligten Operationspflegekräften signifikant weniger beteiligtes Personal $(p=0,031)$ im septi- schen Operationssaal als im Non-COVIDSaal. Die Anzahl der durchschnittlich beteiligten Anästhesisten zeigte sich mit 1,2 bei Patienten mit COVID-19 im Vergleich zu 1,3 bei Non-COVID-Patienten nicht signifikant verändert. Die Anzahl des beteiligten Anästhesiepflegepersonal hingegen zeigte sich bei COVID-19-Patienten mit 1,5 deutlich erhöht $1,0(p=0,022)$.

Bei den vier betrachteten Eingriffen zeigten sich keine signifikanten Unterschiede der Schnitt-Naht-Zeit. Bei Betrachtung der Vorbereitungszeit zeigte sich lediglich bei der Hüft-TEP-Implantation eine signifikante Verlängerung der Vorbereitungszeit bei COVID-19-Patienten mit durchschnittlich 102,3 min vs. 77,8 min bei Non-COVID-Patienten $(p=0,037)$. Eine signifikant verlängerte Gesamtoperationsdauer (Beginn der Operationsvorbereitung im Saal bis Ausschleusen aus Saal) zeigte sich bei PFN-A-Eingriffen bei COVID-19Patienten mit $212,8 \mathrm{~min}$ vs. $142,6 \mathrm{~min}$ bei Non-COVID-Patienten $(p=0,052)$, bei 2 der 4 Patienten mit diesen Eingriffen fand eine postoperative Überwachung im Operationssaal statt.

Bei Betrachtung der direkten postoperativen Phase zeigte sich, dass viele Patienten, die einen der vier betrachteten Eingriffe erhielten, entweder postoperativ auf Intensivstation verlegt wurden (6/13) oder der Eingriff in Lokalanästhesie durchgeführte wurde (5/13), sodass oftmals postoperativ keine längere Überwachung im Operationssaal notwendig wurde.

\section{Diskussion}

Der Beobachtungszeitraum fällt beim Abgleich der Infektionszahlen in Deutschland in den Zeitraum der zweiten COVID-19Welle. Die deutschlandweiten Infektionszahlen des Robert Koch-Instituts stellen sich im graphischen Vergleich kongruent mit dem Patientenaufkommen am Uniklinikum Augsburg dar [3]. Die Anzahl der durchgeführten Operationen in den betrachteten Kalenderwochen zeigt einen klaren Anstieg. Ein vermehrter organisatorischer Aufwand durch spezielle Operationseinheiten, ein erhöhter Personalbedarf im Pflegebereich und ein erhöhter Bedarf postoperativer Überwachung (54\%) auf der Intensivstation sowie viele Eingriffe in Regionalverfahren (38\%) zur Reduktion 
Tab. 3 Vergleich der vier am häufigsten durchgeführten Operationen bei COVID-19- und bei Non-COVID-19-Patienten

\begin{tabular}{|l|l|l|l|l|l|l|l|l|}
\hline & \multicolumn{9}{|l|}{ Gesamt } & \multicolumn{2}{l|}{$\begin{array}{l}\text { COVID-19-Pati- } \\
\text { enten }\end{array}$} & \multicolumn{2}{l|}{$\begin{array}{l}\text { Non-COVID-19- } \\
\text { Patienten }\end{array}$} & $p$-Wert \\
\cline { 2 - 10 } & $(n)$ & $(\%)$ & $(n)$ & $(\%)$ & $(n)$ & $(\%)$ & \\
\hline Einbezogene Patienten & 76 & 100 & 13 & 17 & 63 & 83 & - \\
\hline Alter & - & - & $82,2 \pm 6,8$ & $81,0 \pm 11,0$ & - \\
\hline Geschlecht (m/w) & - & - & $4 / 9$ & $30,8 / 69,2$ & \multicolumn{2}{|l|}{$37 / 26$} & $58,7 / 41,3$ & $0,584^{\text {a }}$ \\
\hline
\end{tabular}

Dringlichkeit

\begin{tabular}{|l|l|l|l|l|l|l|l|}
\hline Elektiv & - & - & 5 & 38,5 & 20 & 32 & $0,748^{c}$ \\
\hline Dringend $<24 \mathrm{~h}$ & - & - & 8 & 61,5 & 40 & 63 & $1,0^{c}$ \\
\hline Notfall & - & - & 0 & 0,0 & 3 & 5 & $1,0^{c}$ \\
\hline
\end{tabular}

Anästhesieverfahren

\begin{tabular}{|l|l|l|l|l|l|l|l|}
\hline Intubationsnarkose & - & - & 7 & 53,8 & 33 & 52 & $1,0^{c}$ \\
\hline Larynxmaskenanästhesie & - & - & 1 & 7,7 & 5 & 8 & $1,0^{c}$ \\
\hline Regional/Lokal & - & - & 5 & 38,5 & 25 & 40 & $1,0^{c}$
\end{tabular}

Operationsdaten

Vorbereitung $(\mathrm{min})$

\begin{tabular}{|l|l|l|l|l|l|}
\hline Embolektomie & - & - & $51,3 \pm 8,1$ & $68,0 \pm 24,0$ & $0,307^{\mathrm{b}}$ \\
\hline $\begin{array}{l}\text { Pertrochantärer Femurnagel } \\
\text { Antirotation }\end{array}$ & - & - & $108,8 \pm 50,9$ & $80,1 \pm 33,1$ & $0,239^{\mathrm{b}}$ \\
\hline Herzschrittmacher & - & - & $31,0 \pm 12,5$ & $41,8 \pm 15,2$ & $0,213^{\mathrm{b}}$ \\
\hline Hüfttotalendoprothese & - & - & $102,3 \pm 4,0$ & $77,8 \pm 25,5$ & $0,037^{\mathrm{b}}$ \\
\hline
\end{tabular}

Schnitt-Naht-Zeit (min)

\begin{tabular}{|l|l|l|l|l|l|}
\hline Embolektomie & - & - & $65,3 \pm 29,5$ & $58,9 \pm 31,0$ & $0,540^{\mathrm{b}}$ \\
\hline $\begin{array}{l}\text { Pertrochantärer Femurnagel } \\
\text { Antirotation }\end{array}$ & - & - & $41,8 \pm 5,1$ & $41,7 \pm 20,5$ & $0,371^{\mathrm{b}}$ \\
\hline Herzschrittmacher & - & - & $29,0 \pm 2,6$ & $36,5 \pm 11,1$ & $0,148^{\mathrm{b}}$ \\
\hline Hüfttotalendoprothese & - & - & $57,0 \pm 13,7$ & $69,5 \pm 17,5$ & $0,225^{\mathrm{b}}$ \\
\hline
\end{tabular}

Gesamtdauer (min)

\begin{tabular}{|l|l|l|l|l|l|}
\hline Embolektomie & - & - & $125,3 \pm 35,0$ & $145,8 \pm 50,8$ & $0,682^{\mathrm{b}}$ \\
\hline $\begin{array}{l}\text { Pertrochantärer Femurnagel } \\
\text { Antirotation }\end{array}$ & - & - & $212,8 \pm 55,9$ & $142,6 \pm 55,8$ & $0,052^{\mathrm{b}}$ \\
\hline Herzschrittmacher & - & - & $76,7 \pm 10,4$ & $81,8 \pm 27,9$ & $0,547^{\mathrm{b}}$ \\
\hline Hüfttotalendoprothese & - & - & $175,0 \pm 18,0$ & $164,2 \pm 41,8$ & $0,382^{\mathrm{b}}$ \\
\hline
\end{tabular}

Beteiligte Personen

\begin{tabular}{|l|l|l|l|}
\hline Operative Ärzte & $1,9 \pm 0,7$ & $1,8 \pm 0,6$ & $1,0^{\mathrm{b}}$ \\
\hline Operatives Pflegepersonal & $2,2 \pm 0,6$ & $2,7 \pm 0,8$ & $0,031^{\mathrm{b}}$ \\
\hline Anästhesiologische Ärzte & $1,2 \pm 0,4$ & $1,3 \pm 0,8$ & $0,612^{\mathrm{b}}$ \\
\hline Anästhesiepflegepersonal & $1,5 \pm 0,7$ & $1,0 \pm 0,6$ & $0,022^{\mathrm{b}}$ \\
\hline Sonstige & $0,3 \pm 0,5$ & $0,5 \pm 0,6$ & $0,29^{\mathrm{b}}$ \\
\hline
\end{tabular}

COVID-19 "Coronavirus disease 2019"

${ }^{\mathrm{a}} \mathrm{X}$ 2-Test

'Wilcoxon-Mann-Whitney-Test

'Fisher's exact test

der postoperativen Überwachung bei den am häufigsten durchgeführten Eingriffen konnte aufgezeigt werden.

Das betrachtete Patientenkollektiv entspricht in Bezug auf Alter, Geschlecht, BMI und Vorerkrankungen dem von Karagiannidis et al. [12] beschriebenen Patientenkollektiv in Deutschland während der ers- ten COVID-19-Erkrankungswelle im Frühjahr 2020. Das mediane Alter betrug in diesem Kollektiv 73 Jahre, beide Geschlechter waren gleich verteilt, bei $17 \%$ wurde eine Beatmung nötig und die Letalität betrug $22 \%$. Als häufige Vorerkrankungen wurden arteriell Hypertonie, Diabetes mellitus und Niereninsuffizient beschrieben. Das hier betrachtete operativ versorgte Patientenkollektiv kann somit als repräsentatives Kollektiv der behandelten COVID-19Patienten betrachtet werden [12].

Die meisten durchgeführten Eingriffe verzeichnete im beobachteten Zeitraum die Klinik für Unfallchirurgie mit einem Anteil von $45 \%$ der durchgeführten Eingriffe. Im Vergleich dazu führte die Abteilung für Allgemein-, Viszeral- und Transplantationschirurgie abseits der COVID-19Pandemie im Jahr 2015 laut Krankenhausreport von 2018 [15] mit 29,1\% die meisten Operationen durch. Dies betrifft zum großen Teil elektiv geplante Eingriffe mit reduzierter Dringlichkeit, welche zum momentanen Zeitpunkt bei SARS-CoV-2positiven Patienten aufgeschoben [8] werden und nach überstandener COVID-19Erkrankung geplant durchgeführt werden $[9,13]$. Im Krankenhausreport entfielen $18 \%$ der Eingriffe auf Orthopädie und Unfallchirurgie und 2,7\% auf gefäßchirurgische Eingriffe. Beim hier betrachteten Kollektiv der COVID-19-Patienten entfielen 25,4\% auf gefäßchirurgische Eingriffe. Dies wird durch die Dringlichkeit der Eingriffe begründet. Bei den durchgeführten unfallchirurgischen Eingriffen handelte es sich meist um dringlich indizierte osteosynthetische Eingriffe oder rezidivierende Weichteileingriffe im Rahmen bestehender Vorerkrankungen der COVID-19-Patienten. Die durchgeführten gefäßchirurgischen Prozeduren bei COVID-19-Patienten fanden im Rahmen dringlicher Indikation bei Extremitätenischämien (Embolektomie) oder bei dringlich indizierten Major- und Minoramputationen sowie im Rahmen chronischer Wundbehandlungen statt. Es zeigt sich somit selten eine Operationsindikation durch die COVID-19-Erkrankung selbst oder damit verbundene Komplikationen, sondern vielmehr eine COVID-19unabhängige Notwendigkeit zur Operation mit paralleler Infektion im Rahmen nosokomialer Ausbrüche oder die dringliche Traumaversorgung bei möglicher Infektion und fehlendem PCR-Test oder angeordneter Quarantäne. Auch die sehr hoch erscheinende Anzahl an Herzschrittmacherimplantationen ist nicht durch die COVID-19-Erkrankung erklärt. Bei einem Patienten handelte es sich um eine Kontaktperson, bei den drei weiteren $\mathrm{Pa}$ tienten handelte es sich um Patienten mit 


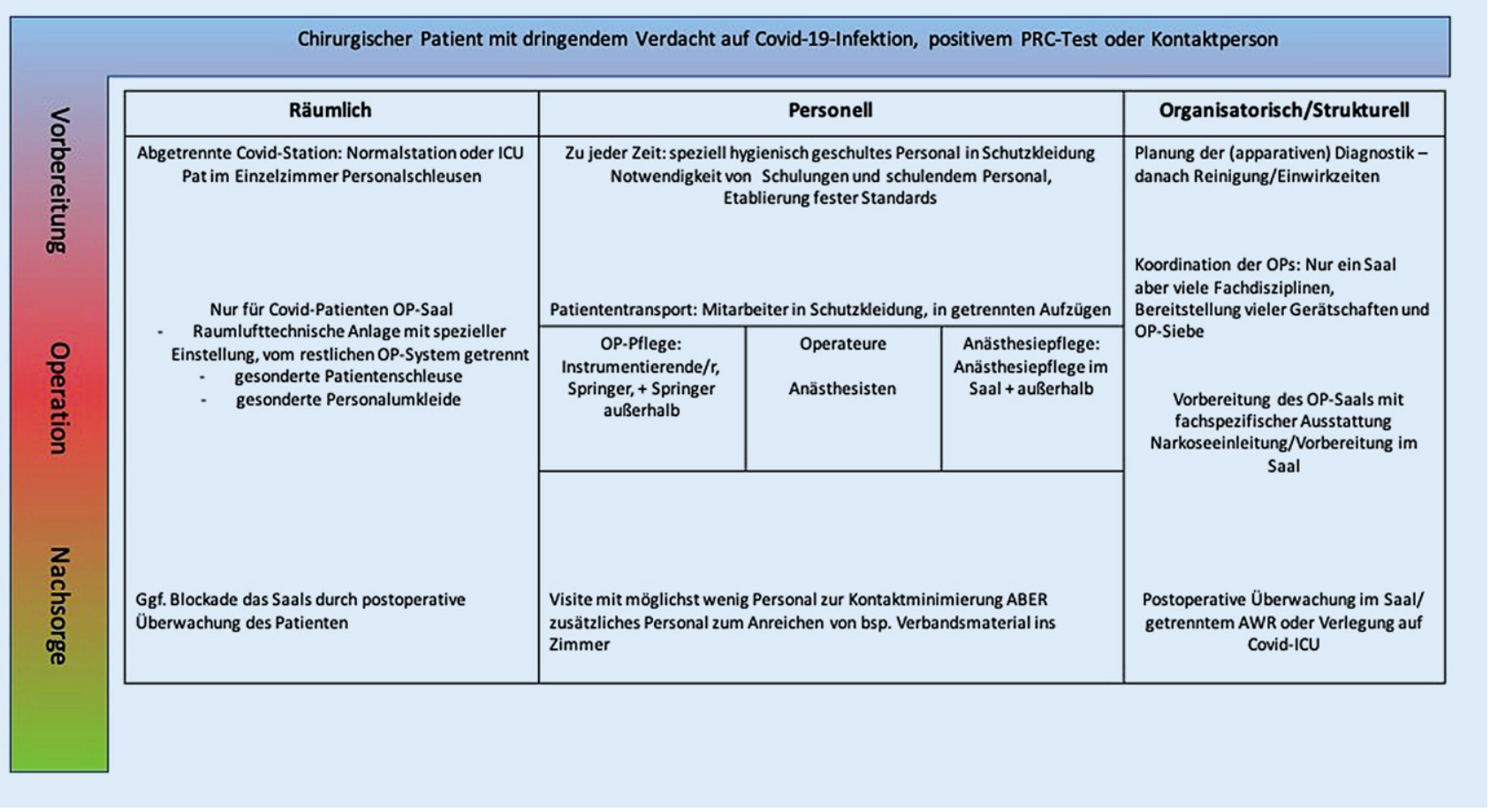

Abb. $3 \Delta$ Organigramm zur Betrachtung zusätzlich zu berücksichtigender Aspekte bei der operativen Versorgung von COVID-19-Patienten. AWR Aufwachraum, COVID "coronavirus disease“, ICU "intensive care unit", OP Operation

höhergradigen Herzrhythmusstörungen und Indikation zur Schrittmacherimplantation, welche präoperativ positiv getestet wurden, allerdings zu diesem Zeitpunkt keine symptomatische COVID-19-Erkrankung aufwiesen.

Im Vergleich zu den Non-COVID-Patienten zeigte die Wahl des Anästhesieverfahrens keinen signifikanten Unterschied zwischen COVID-19-Patienten und NonCOVID-Patienten. Entgegen der Erwartungen wurde nahezu mit gleicher Häufigkeit ein lokales oder allgemeinanästhesiologisches Verfahren gewählt. Dies widerspricht teils den Empfehlungen der Literatur vom Beginn der Pandemie, operative Eingriffe in Allgemeinanästhesie durchzuführen, um die Aerolsolbildung zu minimieren $[14,16]$. Publikationen, inwieweit Patienten wirklich vermehrt in Allgemeinanästhesie operiert wurden, finden sich in der aktuellen Literatur nicht. Nicht außer Acht zu lassen ist, dass besonders die Narkoseeinleitung mit Maskenbeatmung und Intubation ein Risiko zur erhöhten Aerosolbildung und somit ein erhöhtes Infektionsrisiko für das behandelnde Personal darstellt [16]. Es ist also genau abzuwägen, welches der möglichen Anästhesieverfah- ren gewählt wird, um patientenschonend und sicher vorzugehen. Unter den betrachteten Operationen sind Herzschrittmacherimplantation und Embolektomien Eingriffe, die mittels Regionalanästhesieverfahren durchführbar sind. Bei allen betrachteten Operationen an COVID-19-Patienten war ein Anästhesist anwesend, somit auch bei den Operationen, die in Lokal-/ Regionalanästhesie durchgeführt wurden. Dies stellt einen Unterschied zu Operationen bei Non-COVID-Patienten, die in Lokalanästhesie operiert wurden, dar. Dadurch ergibt sich ein im Dokumentationssystem nicht dokumentierter personeller Mehraufwand im ärztlichen Bereich. Ein Beispiel hierfür sind transbrachiale Embolektomien, die bei Non-COVID-Patienten in unserem Klinikum in der Regel ohne Anästhesiebeteiligung in Regionalverfahren durchgeführt werden.

Die Schnitt-Naht-Zeit zeigte keinen wesentlichen Unterschied im Vergleich zwischen COVID-19- und Non-COVID-Patienten in Bezug auf die verglichenen Eingriffe. Dies liegt nach Ansicht der Autoren daran, dass die zu beachtenden Hygienebedingungen die eigentliche Operationszeit nicht gravierend verändern. Die persönli- che Schutzausrüstung bleibt während der Schnitt-Naht-Zeit unverändert und stellt für den Operateur abgesehen von dem zusätzlichen Tragen einer FFP2-Maske keine Veränderung zur Non-COVID-Operation dar. Eine Schutzbrille ist bei operativen Eingriffen aus Sicht der gefäßchirurgischen Autoren generell zu empfehlen $[5,10]$. Eventuelle Wartezeiten auf zusätzliches Material, welches von außerhalb des Operationssaales bereitgestellt werden muss, bringen nur einen minimalen Zeitverlust und werden oft durch den Einsatz erfahrener Operateure ausgeglichen, aber wiederum auch nicht als Mehraufwand im Klinikdokumentationssystem erfasst.

Durch die Nutzung eines Operationssaales für alle Fachabteilungen muss der Operationssaal vor jedem Eingriff mit benötigter fachspezifischer Ausstattung (Röntgengeräte, Laparoskopiezubehör) aufgerüstet werden. Weiteres Material wird in oder vor den Schleusenbereichen bereitgestellt oder bei Bedarf aus dem restlichen Operationssaaltrakt beschafft, was wiederum einen zeitlichen, jedoch nicht nachweisbaren Aufwand bedeutet. Der hierfür benötigte zeitliche Mehr- 
aufwand wird meist durch zusätzliches operatives Pflegepersonal außerhalb des Operationssaales kompensiert, während versucht wird, im Operationssaal die Anzahl der beteiligten Personen möglichst gering zu halten. Dieses Personal außerhalb des Operationssaales wird im Operationsprotokoll nicht erfasst und ging somit nicht in die Analyse des benötigten Personals ein. Nichtsdestotrotz ist hier ein fester und jederzeit telefonisch erreichbarer Ansprechpartner des operativen Pflegepersonals definiert und in der Personalplanung vorgesehen. Dieser Mitarbeiter steht bereit, um Material von außerhalb zu bringen. Dies ist eine mögliche Begründung für die Tatsache, dass im untersuchten Kollektiv signifikant weniger operatives Pflegepersonal bei der Operation von COVID-19-Patienten erfasst wird $(p=0,013)$. Der tatsächliche personelle Mehraufwand wird somit durch die derzeitigen Erfassungssysteme nicht abgebildet und in der Realität ist von einem personellen Mehraufwand auszugehen.

Ein weiterer Faktor, welcher die Gesamtdauer der Operation beeinflusst, ist die Dauer von Naht-Ende bis zum Ausschleusen des Patienten aus dem Operationssaal. Da ein eigener Aufwachraum für COVID-19-Patienten in der analysierten Klinik nicht zur Verfügung steht, verbleibt der Patient zur postoperativen Überwachung so lange wie nötig unter Beobachtung im Operationssaal, bis eine Verlegung auf die COVID-19-Normalstation möglich ist. Patienten die postoperativ auf die Intensivstation verlegt werden, werden direkt dorthin verlegt, sodass dort keine weiteren Verzögerungen entstehen und der Operationssaal schnellstmöglich für die nächste Operation vorbereitet werden kann. Dadurch ist jedoch eine angepasste Kapazität von Intensivbetten vorzuhalten. Diese Handlungsweise entspricht der Empfehlung der Literatur [7]. Die postoperative Überwachung im Operationssaal verlängert die Zeit im Operationssaal und somit die Gesamtoperationsdauer je nach Eingriff. Bei der PFN-A-Implantation zeigte sich ein signifikanter Unterschied, da hier in der Hälfte der Fälle eine postoperative Überwachung im Operationssaal stattfand. Bei den anderen Eingriffen fand häufig eine postoperative Überwachung auf Intensivstation statt bzw. wurden Eingrif- fe in Lokalanästhesie durchgeführt, womit keine verlängerte postoperative Überwachung notwendig wurde. Durch Anwendung regionaler Anästhesieverfahren wurde damit die Gesamtoperationsdauer nicht merklich verlängert und so der Operationssaal schnell wieder zur Verfügung gestellt. Durch die postoperative Verlegung auf Intensivstation ist der Operationssaal wieder schnell verfügbar und auch das operative Anästhesiepersonal steht wieder zeitnah zur Verfügung. Die Vorhaltung und Nutzung eines zusätzlichen Intensivbettes für COVID-19-Patienten stellt allerdings auch einen erheblichen Kostenaufwand dar. Inwieweit dieser Aufwand oder auch die Kosten geringer sind als die Blockade des Operationssaales ist durch diese Untersuchung nicht abgedeckt und sollte im Weiteren mit einer genauen Kostenanalyse untersucht werden. Weiterhin stellt dies nur eine deskriptive Untersuchung der operativen Versorgung von COVID-19-Patienten dar. - Abb. 3 zeigt die perioperativen Abläufe, die eine möglichst reibungslose operative Versorgung ermöglichen. Für eine generelle Betrachtung des Mehraufwandes bei der Versorgung von COVID-19-Patienten ist allerdings eine weitere interdisziplinäre Untersuchung notwendig.

\section{Fazit für die Praxis}

- Die Durchführung von Operationen an COVID-19("coronavirus disease 2019“)Patienten ist in einem sichereren Rahmen ohne zeitliche Verlängerung für die meisten Eingriffe möglich und wird das Gesundheitssystem im Rahmen der Pandemie noch eine weitere Zeit begleiten.

- Um die Patienten bestmöglich zu versorgen und um höchste Sicherheit für das Personal zu gewährleisten, ist ein Bewusstsein für die bestehenden Infektionsrisiken durch regelmäßige Schulungen zu schaffen.

- Der organisatorische sowie personelle und zeitliche Mehraufwand kann durch klinikinterne Ablauforganigramme dargestellt werden. Um einen möglichst reibungslosen Ablauf zu gewährleisten, empfiehlt es sich, diese Punkte präoperativ zu beachten und ggf. durch an die eigenen klinikspezifischen Strukturen angepasste Checklisten oder "standard operating procedures" umzusetzen.

- Die derzeit angewendeten Erfassungssysteme können den Mehraufwand bei Operationen an Patienten mit COVID-19Erkrankungen nicht adäquat abbilden.

\section{Korrespondenzadresse}

\section{H. Kerndl}

Gefäßchirurgie, Medizinische Fakultät,

Universitätsklinikum Augsburg

Stenglinstr. 2, 86156 Augsburg, Deutschland

hagen.kerndl@uk-augsburg.de

\section{Einhaltung ethischer Richtlinien}

Interessenkonflikt. H. Kerndl, D. Liebetrau, S. Zerwes, C. Römmele und A. Hyhlik-Dürr geben an, dass kein Interessenkonflikt besteht.

Für diesen Beitrag wurden von den Autoren keine Studien an Menschen oder Tieren durchgeführt. Für die aufgeführten Studien gelten die jeweils dort angegebenen ethischen Richtlinien.

\section{Literatur}

1. Al-Omar K, Bakkar S, Khasawneh L et al (2020) Resuming elective surgery in the time of COVID-19: a safe and comprehensive strategy. Updates Surg 72:291-295

2. Robert Koch Institut (2021) Empfehlungen des RKI zu Hygienemaßnahmen im Rahmen der Behandlung und Pflege von Patienten mit einer Infektion durch SARS-CoV-2

3. Robert-Koch-Institut (2021) Gesamtübersicht der pro Tag ans RKI übermittelten Fälle, Todesfälle und 7-Tage-Inzidenzen nach Bundesland und Landkreis

4. Anonymous (1996) Gesetzliche Unfallversicherung - §34 Durchführung der Heilbehandlung. In: Siebtes Buch Sozialgesetzbuch. p 1254

5. Berridge DC, Lees TA, Chamberlain J et al (1993) Eye protection for the vascular surgeon. Br J Surg 80:1379-1380

6. Bresler AM, Bischoff MS, Bockler D (2020) SARSCoV-2-How can and must medical personnel protect themselves? Gefässchirurgie. https://doi. org/10.1007/s00772-020-00698-1

7. BrindleME, Gawande A(2020) Managing COVID-19 in surgical systems. Ann Surg 272:e1-e2

8. Collaborative CO (2020) Elective surgery cancellations due to the COVID-19 pandemic: global predictive modelling to inform surgical recovery plans. Br J Surg 107:1440-1449

9. Collaborative CO, Globalsurg C (2021) Timing of surgery following SARS-CoV-2 infection: an international prospective cohort study. Anaesthesia 76:748-758

10. Davies CG, Khan MN, Ghauri ASKet al (2007) Blood and body fluid splashes during surgery - the need for eye protection and masks. Ann R Coll Surg Engl 89:770-772

11. Gmbh FD DIN 1946-4, Ausgabe 2018/09: Raumlufttechnik - Teil 4: Raumlufttechnische Anlage in Gebäuden des Gesundheitswesens. In:

12. Karagiannidis C, Mostert $C$, Hentschker $C$ et al (2020) Case characteristics, resource use, and outcomes of 10021 patients with COVID-19 admitted to 920 German hospitals: an observational study. Lancet Respir Med 8:853-862

13. Lenzen-Schulte M (2021) SARS-CoV-2: Nicht ohne Impfung in den OP-Saal. Dtsch Arztebl Int 118:862

14. Saracoglu KT, Dalkilinc Hokenek U, Saracoglu A et al (2020) COVID-19 patients in the operating room a concise review of existing literature. Minerva 
Anestesiol. https://doi.org/10.23736/S0375-9393. 20.15015-6

15. Spindler J (2018) Operationen und medizinische Prozeduren. Krankenhausreport 2018:418-425

16. Tang LY, Wang J (2020) Anesthesia and COVID-19: what we should know and what we should do. Semin Cardiothorac Vasc Anesth 24:127-137

17. Ti LK, Ang LS, Foong TW et al (2020) What we do when a COVID-19 patient needs an operation: operating room preparation and guidance. Can J Anaesth 67:756-758

\section{Resource requirements in the surgical treatment of COVID-19 patients at a university clinic of maximum care}

Background: Surgical procedures in patients suffering from coronavirus disease 2019 (COVID-19) are possible under strict hygiene and protective measures and are currently carried out regularly. This study examined how much additional work this involves. Material and methods: A structured evaluation of 71 surgical procedures performed at the Augsburg University Hospital between 1 November 2020 until 31 December 2020 was carried out. The operations on COVID-19 patients were compared to procedures on non-COVID-19 patients with respect to temporal, structural and staff resources, exemplified by four interventions: transbrachial embolectomy, total hip arthroplasty (H-TEP), proximal femoral nail antirotation (PFN-A) and new implantations of cardiac pacemakers.

Results: The incision to suture times between the interventions in patients with COVID-19 and non-COVID-19 patients did not show any significant differences in any of the four interventions evaluated. The postoperative monitoring in the operating room, which is identified as time-consuming in many interventions, was often circumvented by postoperative transfer to the intensive care unit or by the use of local anesthetic procedures. For major operations, such as H-TEP, the preparation time was shown to be significantly longer $(p=0.037)$. Furthermore, there was a significantly higher requirement for anesthesia nursing personnel of 1.5 vs. $1.0(p=0.02)$.

Conclusion: A quantification of the additional effort of operative treatment is difficult due to the already complex care of COVID-19 patients; however, it can be assumed that there is an increased need for additional human and structural resources due to the supply of material from outside the operating room, which is not documented in the standard recording.

\section{Keywords}

Operation management · Transbrachial embolectomy · Total hip arthroplasty · Proximal femoral nail antirotation - Cardiac pacemaker 